\title{
Channel Estimation and Data Transmission in Massive MIMO TDD Systems
}

\author{
Youjie Kang1, Tianyang Lu² \\ ${ }^{1}$ Beijing University of Technology, Beijing, China \\ ${ }^{2}$ Sun Yat-sen University, Guangzhou, China \\ Email: cis2018@foxmail.com
}

How to cite this paper: Kang, Y.J. and $\mathrm{Lu}$, T.Y. (2019) Channel Estimation and Data Transmission in Massive MIMO TDD Systems. Int. J. Communications, Network and System Sciences, 12, 170-187. https://doi.org/10.4236/ijcns.2019.1210012

Received: September 2, 2019

Accepted: October 20, 2019

Published: October 23, 2019

Copyright (C 2019 by author(s) and Scientific Research Publishing Inc. This work is licensed under the Creative Commons Attribution-NonCommercial International License (CC BY-NC 4.0). http://creativecommons.org/licenses/by-nc/4.0/ (c) (7) (5) Open Access

\begin{abstract}
This paper mainly elaborates the studies of channel estimation and downlink data transmission in Massive MIMO. As there are different types of interference in single-cell and multi-cell systems, this paper establishes different models for them separately. In terms of uplink training, for getting channel state information, we introduce LS and MMSE channel estimation algorithms and make a comparison between them. At the same time, the problem of pilot contamination is solved by cell classification and pilot identification. Next, this paper defines mathematical models for downlink data transmission. We use pre-coding methods (including Zero-forcing and Maximal Ratio Combining schemes) and optimize power distribution to improve channel capacity and transmission rate. Furthermore, this paper provides numerical results to show the simulation performance in both single-cell and multi-cell systems and extends to prospects in the future.
\end{abstract}

\section{Keywords}

Channel Estimation, Pilot Contamination, Downlink Data Transmission, Pre-Coding Schemes, Power Distribution

\section{Introduction}

MIMO (Multiple-Input Multiple-Output) refers to using of multiple transmitted and received antennas at the transmitted and received sides to transmit and receive signals. It can make full use of space resources, so the channel capacity and communication quality can be increased without increasing spectrum resources and antenna transmission power. Massive MIMO system is generally considered to be proposed by Marzetta T. L. of Bell Labs in 2010 [1]. It is the MIMO system whose number of antennas in the base station is order of magnitude larger than 
the number of the users. By increasing the number of antennas at the base station, we can average out the effects of fading, thermal noise, intra-cell interference and improve the transmission rate. Therefore, the channel capacity will be larger and the quality of the channel will be greatly ameliorated. Mostly, Massive MIMO uses TDD technology rather than FDD [2]. It makes the uplink and downlink of the channel use the same frequency band but different time slots. Assuming channel reciprocity, the uplink and downlink channel matrix are the same. So it is easy to estimate the channel.

Nowadays the MIMO technology has become one of the key technologies in the field of wireless communication [3]. Through continuous development in recent years, MIMO technology will be more and more widely applied to various wireless communication systems. In terms of wireless broadband mobile communication systems, the 3rd Generation Partnership Project (3 GPP) has added MIMO technology-related content to the standards, and MIMO technology will also be applied to $\mathrm{B} 3 \mathrm{G}$ and $4 \mathrm{G}$ systems. In wireless broadband access systems, standards such as $802.16 \mathrm{e}, 802.11 \mathrm{n}$, and 802.20 , which are under development, also employ MIMO technology. The Massive MIMO technology is not yet widely used today. However, in the field of 5G wireless communication, Massive MIMO wireless communication will utilize more antennas to transmit and receive information in the future [4]. The power utilization rate and system spectrum utilization rate will significantly improve. It will lay a solid foundation for $5 \mathrm{G}$ to achieve faster and more stable targets. Because of these obvious advantages, the Massive MIMO is regarded as the key technology to 5G communication.

\subsection{Motivation}

Massive MIMO has been a hot topic in recent years, since it provides rich spatial freedom and more possible arrival path, which can improve the capacity and reliability of wireless systems. And some researchers also find that it significantly improves system spectral efficiency and energy efficiency [5]. The benefits of multiple antennas, especially at the base station, are not limited.

When the base station has a large number of antennas, the phase of the transmitted signal can be adjusted to form superimposed signals at the receiving antenna, which make each transmitted antenna only needs to transmit signals with low power. By constructing different beams directed to multiple target users, interference between the beams can be effectively reduced. Moreover, based on channel reciprocity, the cost of pilot training is independent to the number of base station antennas but only related to the number of users per cell. So when the number of base station antennas tends to infinity, the feedback overhead of the system is not increased. Perfectly matched to Massive MIMO is millimeter wave which is another key technology of 5G [6]. Millimeter waves have a wide bandwidth, but the attenuation is strong. The beamforming of Massive MIMO just complements the short board. If the antenna size we use is fixed relative to the infinite wavelength, the increase in carrier frequency means that the antenna 
becomes smaller and smaller, that is, in the same space, we can plug in more antennas. Based on this fact, we can compensate for high-frequency path loss by increasing the number of antennas without increasing the antenna size.

\subsection{Aims}

Massive MIMO is recognized as one of the key technologies of 5G mobile communication systems, which can meet the needs of high capacity, high spectral efficiency and high sum rates. There have been many studies on multi-user MIMO in recent years, but few perfect multi-cell systems with good solutions for both channel estimation and transmission contamination have been displayed. The literatures [7] and [8] introduce some methods for pilot contamination in the process of uplink training like shifting the location of pilots in time frames used in neighboring cells and using non-orthogonal training sequences. [9] and [10] propose some solutions to reduce downlink interference including using a special multi-cell MMSE-based precoding scheme and derive optimized pre-conditioning matrix. In our paper, we are committed to applying Massive MIMO to multi-cell cellular systems and make sure that each user in each cell can perform stable and fast wireless communication with the base station of the cell in which it is located. We also compare the performances when using different training pilots and pre-coding schemes in both uplink and downlink transmission and proposes some new methods. As well as this, we focus on power distribution, which can be used to reduce the interference to some degrees.

We aim to overcome the pilot contamination problem in uplink channel estimation and reduce the interference from other base stations when downlink data is transmitted to each user, so that this multi-cell communication system has great communication quality to satisfy enough users.

\subsection{Organization}

The rest of this paper is organized as follows. In Section 2, the problem statement is displayed, which leads to description of the system model with some assumptions and mathematical formulas. We present the analysis and approaches of the whole design process in Section 3, which includes methods and optimized designs for channel estimation and data phase completely. Then, we present simulation results in Section 4 and mention important findings in Section 5. After that, we finally talk about the prospects of the future work and provide a conclusion in Section 6, which is followed by an acknowledgement at last.

\section{Model Construction}

\subsection{Problem Statement}

\section{1) Pilot contamination in uplink training}

The good performance of Massive MIMO system depends on that the base station can estimate the channel state information (CSI), but the noise received by the antenna will affect the channel estimation. Because channel coherence in- 
terval is short, not all cell users can transmit mutually orthogonal pilot sequences. Therefore, pilots need to be multiplexed, which results in channel estimation being interfered by other cells using the same pilot. Pilot contamination seriously restricts the performance of Massive MIMO multi-cell TDD systems and has become an urgent problem to be solved in communication systems.

\section{2) Common channel interference in downlink transmission}

In downlink transmission, the base stations send signals to multiple users at the same time. The user receives much interference from information to other users in addition to the signals that he wishes to receive. Therefore, in the downlink of a multi-user MIMO system, it is necessary to eliminate such interference.

\subsection{System Model}

\subsubsection{Assumption}

We aim to consider single-cell system model first, and adjust to multi-cell system model with some methods which help us to reduce the inter-cell interference. Some basic assumptions we make are shown below:

1) Each cell covers a range of circle with a radius of 2000 meters;

2) Users are distributed at various locations in each cell with the same probability;

3) For each base station (user), we only consider the impact of information sent by users (base stations) within $10 \mathrm{~km}$;

4) The path loss $(\beta)$ of the channel depends on the distance between the user and the base station, which is regarded as large scale attenuation and is known in the system;

5) The small scale fading factor $(h)$ is independent identically distributed: zero-mean, circularly-symmetric complex Gaussian $(0,1)$ random distribution. The system doesn't know these factors, which means that the channel state information (CSI) should be estimated at each base station;

6) Some notations are shown in Figure 1.

\subsubsection{Single-Cell System Model}

The single-cell system model consists of a base station with $M$ antennas and $K$ single-antenna users $(M \gg K)$.

1) Uplink training for channel estimation

\begin{tabular}{|c|c|}
\hline $\mathrm{h}_{\mathrm{k}}^{*}$ & Complex conjugate transpose of $\mathrm{h}_{\mathrm{k}}$ \\
\hline $\mathrm{M}$ & The number of antennas in a cell \\
\hline $\mathrm{K}$ & The number of users in a cell \\
\hline $\mathrm{h}$ & Small scale fading factor \\
\hline$\beta$ & Large scale fading factor (path loss) \\
\hline$\sigma_{\mathrm{w}}^{2}$ & Noise factor \\
\hline
\end{tabular}

Figure 1. Notation. 
As $M \gg K$, smaller number of pilots are required in uplink channel estimation. So in order to reduce the complexity of system, we ask users to send uplink training pilots and do the channel estimation at the base station. The received signal under noise can be expressed as:

$$
\boldsymbol{Y}=\sqrt{P_{U} T_{U}} \boldsymbol{H} \boldsymbol{X}+\boldsymbol{W}
$$

\section{2) Downlink data transmission}

We first consider about the received signal at user $\mathrm{k}$ without interference from other users. The path loss factor $(\beta)$ and small scale fading factor $(h)$ are described above (Denote this channel coefficient $(h)$ from the k-th user in the single-cell system to the $i$-th antenna of the Base station as $\left.h_{k i}\right)$. We use $p_{k}$ to represent the downlink power from base station to user $k$. The equation is shown below:

$$
y_{k}=\sum_{i=1}^{M} \sqrt{P_{k} \cdot \beta_{k}} \cdot h_{k i} \cdot x_{k}+w_{k}
$$

Then, consider the situation that each signal from the base station can pass through the channel between base station and user $k$. We need pre-coding to reduce this kind of interference at base station. We use $v_{k}$ to denote the pre-coding vector for user $k$. The received signal $y_{k}$ can be represented as:

$$
y_{k}=\sum_{i=1}^{K} \sqrt{P_{f} \cdot \beta_{k}} \cdot\left[\begin{array}{llll}
h_{k 1} & h_{k 2} & \cdots & h_{k M}
\end{array}\right] \cdot v_{i} \cdot x_{i}+w_{k}
$$

\subsubsection{Multi-Cell System Model}

The multi-cell system model consists of $L$ cells. Each cell is made up of a base station with $M$ antennas and $K$ single-antenna users as discussed above. As shown in Figure 2 ( 2 cells system).

\section{1) Uplink training for channel estimation}

The pilot signal received by the base station in 1 cell is expressed as

$$
\boldsymbol{Y}_{l}=\left(\boldsymbol{H}_{1} \boldsymbol{X}_{1}+\boldsymbol{H}_{2} \boldsymbol{X}_{2}+\cdots+\boldsymbol{W}\right)
$$

We can find that it contains the pilot from users in other cells. As the same pilot is used in different cells, the contamination pilot cannot be avoided. We will give solutions to reduce pilot contamination impact in next section.

\section{2) Downlink data transmission}

In this case, we should consider about the interference from other cells. The signal received by $k$ - $t$ th user in the $l$-th model can be represented as:

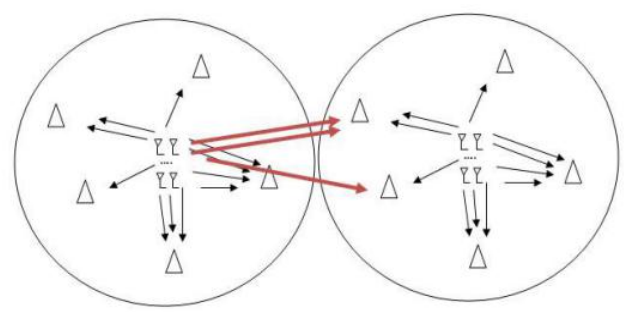

Figure 2. 2 cells system model. 


$$
y_{l k}=\sum_{j=1}^{L} \sum_{i=1}^{K} \sqrt{P_{f} \cdot \beta_{j l k}} \cdot\left[\begin{array}{llll}
h_{j 1 l k} & h_{j 2 l k} & \cdots & h_{j M l k}
\end{array}\right] \cdot v_{j i} \cdot x_{j i}+w_{l k}
$$

\section{Approach}

\subsection{Channel Estimation}

Channel estimation is a very important part in wireless transmission, because the accuracy of the estimation will impact the reality and capacity of the data transmission. This part is about how to do the channel estimation and the way to deal with the pilot contamination.

\subsubsection{Downlink or Uplink}

Since the channel reciprocity, training pilots can be sent in downlink or uplink. However, in order to reduce the complexity of estimation, we must make a choice between them. If we do the estimation in downlink:

$$
\begin{gathered}
\boldsymbol{Y}=\boldsymbol{H}^{*} \boldsymbol{X}+\boldsymbol{W} \\
\boldsymbol{H}=\left(\boldsymbol{X} \boldsymbol{X}^{*}\right)^{-1} \boldsymbol{X} \boldsymbol{Y}^{*}
\end{gathered}
$$

where $\boldsymbol{X} \in C^{M \times T_{D}}$ is the matrix containing $T_{D}$ pilots transmitted from $M$ antennas and $\boldsymbol{Y} \in C^{K \times T_{D}}$ is the received signal matrix on $K$ Users. In order to make $\boldsymbol{X} \boldsymbol{X}^{*}$ reversible, we must let $T_{D} \geq M$, so the pilots matrix will be very huge because of the large number of antennas on BS, this will cause a huge complexity for calculating $\left(\boldsymbol{X} \boldsymbol{X}^{*}\right)^{-1}$. Because the estimation is done on users' part, BS needs a feedback to get the channel. But, if we do the estimation in uplink:

$$
\begin{gathered}
\boldsymbol{Y}=\boldsymbol{H} \boldsymbol{X}+\boldsymbol{W} \\
\boldsymbol{H}=\boldsymbol{Y} \boldsymbol{X}^{*}\left(\boldsymbol{X} \boldsymbol{X}^{*}\right)^{-1}
\end{gathered}
$$

$\boldsymbol{X} \in C^{K \times T_{U}}$ is the matrix containing $T_{U}$ pilots transmitted from $K$ Users.

$\boldsymbol{Y} \in C^{M \times T_{U}}$ is the received signal matrix on BS antennas array.

In order to make $\boldsymbol{X} \boldsymbol{X}^{*}$ reversible, we must let $T_{U} \geq K$. This time, the size of pilot matrix only depends on the number of Users, so the calculation is much easier. And because the estimation is done on BS, it doesn't need a feedback in the system. Obviously, doing the estimation in uplink is superior to do that in downlink.

\subsubsection{LS (Least Square) and MMSE (Minimum Mean Square Error)}

The channel estimation algorithms also have impact on the performance of channel estimation. LS and MMSE are two common algorithms used in estimation, but the target of them are different.

After choosing to do the estimation in uplink, we have the received uplink signal under noise:

$$
\boldsymbol{Y}=\sqrt{P_{U} T_{U}} \boldsymbol{H} \boldsymbol{X}+\boldsymbol{W}
$$

As for LS, it wants to minimize the squared error $\left\|\boldsymbol{Y}-\sqrt{P_{U} T_{U}} \boldsymbol{H} \boldsymbol{X}\right\|^{2}$, and we can solve this problem by: 


$$
\boldsymbol{H}_{\mathrm{LS}}=\boldsymbol{Y} \boldsymbol{X}^{*}\left(\boldsymbol{X} \boldsymbol{X}^{*}\right)^{-1} / \sqrt{P_{U} T_{U}}
$$

The channel estimation algorithm based on the LS criterion has a simple structure, and the calculation complexity is small only by performing one division operation [11]. However, the influence of noise is neglected, but the channel estimation is sensitive to the noise influence. So when the channel noise is large, the accuracy will greatly reduce.

For MMSE, it wants to find a $\boldsymbol{G}$ that make $E\left\{\boldsymbol{Y}^{*}\left(\boldsymbol{Y G}-\sqrt{P_{U} T_{U}} \boldsymbol{H}\right)\right\}=0$, in order to minimize the mean square error, so we have:

$$
\boldsymbol{H}_{\mathrm{MMSE}}=\boldsymbol{Y}\left(P_{U} T_{U} \boldsymbol{X}^{*} \boldsymbol{D} \boldsymbol{X}+\sigma_{w}^{2} \boldsymbol{I}\right)^{-1} \sqrt{P_{U} T_{U}} \boldsymbol{X}^{*} \boldsymbol{D}
$$

MMSE considers the influence of noise when performing the optimization solution, so the mean square error of the channel estimation is small. However, since we have to calculate the inverse operation of the matrix when using MMSE channel estimation, when the number of users $K$ increases, the calculation will also have a high complexity.

\subsubsection{Pilot Contamination}

In only one cell, the error of estimation comes from the added noise of interferences among users can be solved by orthogonal pilots. Increasing the uplink power is a way to deal with the noise, however, there is usually a power limit in the transmission, and we cannot initially increase the power. So under the power limit, we choose to increase the length of pilot $T_{U}$. It is a way that sacrificing time in exchange for accuracy, when the pilot is designed longer, it will have more power which reduce the affection of noise.

But in multi-cell model, the BS will receive pilots from the users in other cells. When pilots are multiplexed in all cells, BS can't discriminate these pilots, so there will be a very serious Users interference (see from Figure 3 ).

Now we have the received uplink signal under noise:

$$
\boldsymbol{Y}=\left(\boldsymbol{H}_{1} \boldsymbol{X}_{1}+\boldsymbol{H}_{2} \boldsymbol{X}_{2}+\cdots+\boldsymbol{W}\right)
$$

We can know from the system model that the fading parameter $\beta$ rapidly decreases as the spatial distance increases. Therefore, the most serious contamination always comes from neighboring cell of the target cell. If their pilot emissions can be staggered from each other, it is possible to improve the performance of the entire system. Reference proposes a method of aligning cell pilot transmission time slots to data transmission time slots of other cells in order to reduce pilot contamination of the entire system. But in a large-scale multi-cell system, it is obviously too ideal to make the pilot transmission time slots of the target cell always align to the data transmission time slots of other cells [12] [13]. So we think of dividing the cells into three types and design different pilots for them. As shown in Figure 4.

In method one, other two types of cells will keep silent when one type of cells are sending pilots (See from Figure 5). 
In method two, three kinds of pilot are orthogonal to each other (see from Figure 6).

Now, when BS is receiving pilots, it will only be interfered by cells in same type. Since the cells in same type are far away from each other, the interference is much lower.

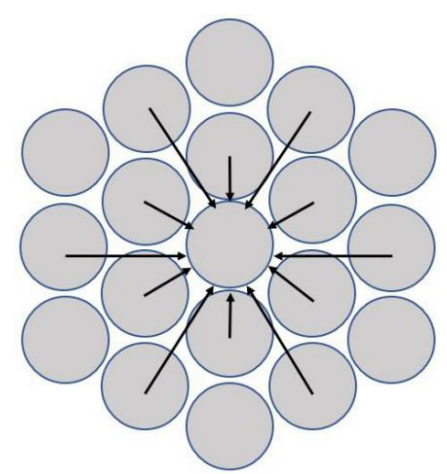

Figure 3. Multi-cell model (1).

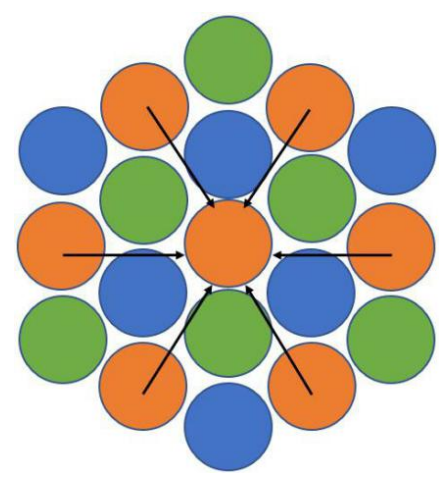

Figure 4. Multi-cell model (2).

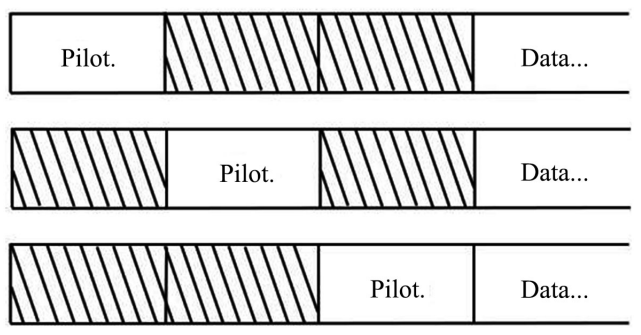

Figure 5. Method 1.

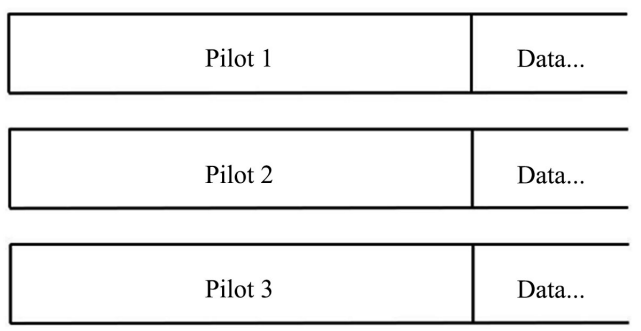

Figure 6. Method 2. 


\subsection{Downlink Data Transmission}

After estimating the channel at the base station, each component in the cell knows the channel condition. Basically, the channels are full duplex so that there are both uplink and downlink transmission in the system. As for uplink data transmission, we can do some useful processes and combining at the base station to separate information from different users reliably and accurately. While for downlink data transmission, we cannot require users to reduce the interference any more. Hence it's more complicated to have good performance at downlink transmission and that is what we focus on in this paper.

\subsubsection{Problem Review}

Like what we mention in Section 3 and Section 4, there will be intra-cell interference in the system, which means that a specific user will receive a mixed signal consisted of information components for all the other users in his cell. As well as this, inter-cell interference from other cells will also have influences in multi-cell system.

\subsubsection{Mathematical Representation}

In this part, we use mathematical equation to represent the signals a particular user receives.

1) Received signal at $k$-th user in a single cell (with intra-cell interference):

$$
y_{k}=\sum_{i=1}^{K} \sqrt{P_{f} \cdot \beta_{k}} \cdot\left[\begin{array}{llll}
h_{k 1} & h_{k 2} & \cdots & h_{k M}
\end{array}\right] \cdot v_{i} \cdot x_{i}+w_{k}
$$

2) Received signal at $k$-th user in 1 -th cell (with intra-cell interference and inter-cell interference):

$$
y_{k}=\sum_{j=1}^{L} \sum_{i=1}^{K} \sqrt{P_{f} \cdot \beta_{j l k}} \cdot\left[\begin{array}{llll}
h_{j 11 k} & h_{j 2 l k} & \cdots & h_{j M l k}
\end{array}\right] \cdot v_{j i} \cdot x_{j i}+w_{l k}
$$

here, we can clearly see that we consider the influence from signals sent from all base stations to all the users.

So, in order to make the system have a better performance, we should try to do something to improve the SINR. Two methods are provided in the paper and we are going to discuss pre-coding schemes and power distribution separately in detail.

\subsubsection{Pre-Coding Schemes}

Here, we give two pre-coding schemes for downlink transmission (Zero-forcing and Maximal Ratio Combining). Comparing the different goals, zero-forcing [14] aims to cancel inter-user interference while MRC helps to maximize the gain toward the specific user [9].

\section{Scheme 1: Zero-forcing}

Subject to the conditions below:

$$
\begin{gathered}
\text { Maximum }\left|h_{k}^{*} \cdot v_{k}\right|^{2} \\
h_{j}^{*} \cdot v_{k}=0, \quad j \neq k \&\left\|v_{k}\right\|=1
\end{gathered}
$$


In order to represent it clearly, we define matrix $\boldsymbol{G}$ :

$$
\boldsymbol{G}=\boldsymbol{H}\left(\boldsymbol{H}^{*} \boldsymbol{H}\right)^{-1}
$$

Hence, pre-coding vector $v$ can be written as form:

$$
v_{k}=\frac{G_{:, k}}{\left\|G_{:, k}\right\|}
$$

In this case, SINR for $U E-k$ is

$$
\frac{P_{k}\left|h_{k}^{*} \cdot v_{k}\right|^{2}}{\sigma_{w}^{2}}
$$

\section{Scheme 2: Maximal ratio combining}

Subject to the conditions below:

$$
\begin{gathered}
\text { Maximum }\left|h_{k}^{*} \cdot v_{k}\right|^{2} \\
\text { also }\left\|v_{k}\right\|=1
\end{gathered}
$$

Hence, pre-coding vector $\mathrm{v}$ can be written as form:

$$
v_{k}=\frac{h_{k}}{\left\|h_{k}\right\|}
$$

The basic idea of this method is to maximize the gain of the wanted signal without taking care of the inter-user interference, which can be found in the equation of SINR. As shown below (SINR at $U E-k$ ):

$$
\frac{P_{k} \cdot\left\|h_{k}\right\|^{2}}{h_{k}^{*}\left(\sum_{i=1, i \neq k}^{K} P_{i} \cdot v_{i} \cdot v_{i}^{*}\right) h_{k}+\sigma_{w}^{2}}
$$

\subsubsection{Power Distribution}

The second method for improving the sum rate of the system is power distribution. We will ignore the bandwidth in the equation because all the base station use the same bandwidth in our system, and it doesn't matter when we are looking for the better data rate by changing power distribution. In this case, we focus on transmission with zero-forcing pre-coding.

We represent the sum rate as form:

$$
C_{\text {sum }}=\sum_{n=1}^{K} \log _{2}\left(1+\frac{P_{n} *\left|h_{n}^{*} \cdot v_{n}\right|^{2}}{\sigma_{w}^{2}}\right)
$$

And the system has a power limitation:

$$
\sum_{n=1}^{K} P_{n}=P_{\text {sum }}
$$

Here, we use Lagrange Multiplier Method to find the Optimum condition:

$$
L\left(\lambda, P_{1}, P_{2}, \cdots, P_{K}\right)=\sum_{n=1}^{K} \log _{2}\left(1+\frac{P_{n} *\left|h_{n}^{*} \cdot v_{n}\right|^{2}}{\sigma_{w}^{2}}\right)+\lambda\left(\sum_{n=1}^{K} P_{n}-P_{\text {sum }}\right)
$$


Therefore, we have:

$$
P_{n}(n=1,2, \cdots, K)=\left(\frac{1}{\lambda \ln 2}-\frac{\sigma_{w}^{2}}{\left|h_{n}^{*} \cdot v_{n}\right|^{2}}\right)^{+}
$$

Substituting (23) in (21), we get:

$$
\mu=\frac{1}{\lambda \ln 2}=\frac{P_{\text {sum }}+\sum_{n=1}^{K} \frac{\sigma_{w}^{2}}{\left|h_{n}^{*} \cdot v_{n}\right|^{2}}}{K}
$$

According to Equations (23) and (24), we can find that some users cannot get impact information due to the unbalanced power distribution. In order to handle this problem, we set a power threshold to make sure that the amount of power allocated to each user is enough. We have:

$$
P_{t h}<\frac{P_{\text {sum }}}{K}
$$

We design the algorithm for the system. As shown below (Consider about the power for $U E-k)$ :

$$
\begin{gathered}
\text { If }\left(\frac{1}{\lambda \ln 2}-\frac{\sigma_{w}^{2}}{\left|h_{k}^{*} \cdot v_{k}\right|^{2}}\right)^{+}<P_{t h}, P_{k}=P_{t h} \\
\text { Else } P_{k}=\left(\frac{1}{\lambda \ln 2}-\frac{\sigma_{w}^{2}}{\left|h_{k}^{*} \cdot v_{k}\right|^{2}}\right)^{+}
\end{gathered}
$$

Then, we have a new system model without $U E-k$ and do the similar inductive recursion again:

$$
P_{n}(n=1,2, \cdots, K, n \neq k)=\left(\frac{1}{\lambda_{\text {new }} \ln 2}-\frac{\sigma_{w}^{2}}{\left|h_{n}^{*} \cdot v_{n}\right|^{2}}\right)^{+}
$$

By using this algorithm, we can have such a more balanced system without increasing the total power.

\subsubsection{Analysis for Multi-Cell System}

As for multi-cell model, if we do not perform additional operations at the base stations, the performance of this system may be poor [1]. In order to reduce the inter-cell interference, base stations need to share information of each user and channel characteristics for joint pre-coding.

This kind of pre-coding process is more complicated than that in the single-cell system and that is the reason we try to use a base station controller to help complete it.

In this model, the base station controller provides the target base station with the information about channels in closed cells. And the target base station can do the pre-coding according to this information and make vectors orthogonal. Of course the complexity is much higher than the previous case, and in the fu- 
ture, we will try to figure out if this idea really works.

\section{Results and Analysis}

\subsection{Performance Comparison between LS and MMSE}

We can see from Figure 7 that LS and MMSE performances are better when the uplink power increase. When the uplink power is the same, the estimation error of the MMSE algorithm is smaller than the LS algorithm. The MMSE algorithm improves the system performance better than the LS algorithm. In the low uplink power situation, the difference between LS and MMSE is huge, but when the uplink power is high, their performances are similar. It is because LS algorithm ignores the noise influences when MMSE algorithm reduces the influence.

\subsection{Performance of Increasing Pilot Length}

Figure 8 tells us that the longer pilot performs better than the shorter one, it is because that it has more power to reduce the impact of noise. We can see that in the low power situation, there is an obvious difference between them, but when the power get higher which means the impact of noise is lower, the performance of them are similar.

\subsection{Performance of Pilot Contamination Solving}

In the multi-cell situation, the estimation error is described by Figure 9. When the uplink power gets higher, the interferences even become more serious. It is because when the pilot we want has more power, the pilot from other cells will also be stronger which leads to a serious pilot contamination. However, when we design different pilots for different cells, the contamination only comes from the cells using same pilot which are far away from the target cell. So this method obviously reduces the estimation error.

From Figure 10 we know that Method 2 even works better than Method 1. It is because Method 1 only use 1/3 time of the pilot and keep silence for the other time. However, the pilot in Method 2 sends signal all the time, so it has more power compared to Method 1. In low uplink power situation, this extra power is very important for reducing the influence of noise, so Method 2 has a much better performance. But when the uplink power gets higher, the performance of these two methods will be similar.

\subsection{Performance Comparison between ZF and MRC}

Figure 11 shows the results when using perfect and estimated channels with these two pre-coding methods (Zero-forcing Maximal Ratio Combining). We can find that the curve of estimated channel is closed to that of the perfect channel under the same circumstance, while there is a big difference between using two pre-coding methods. Zero-forcing performs better than Maximal Ratio Combining. It makes sense because the complexity of Zero-forcing is much higher than that of Maximal Ratio Combining. 
We use only the perfect channel to check what happens as the number of antennas increases. As shown in Figure 12, we can find that more antennas result in better SINR as expected and Zero-forcing still performs better than Maximal Ratio Combining.

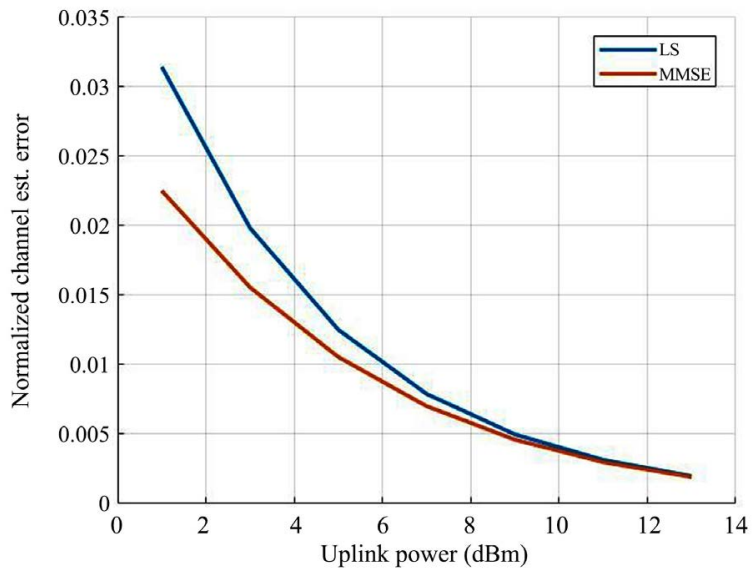

Figure 7. Performance of LS and MMSE.

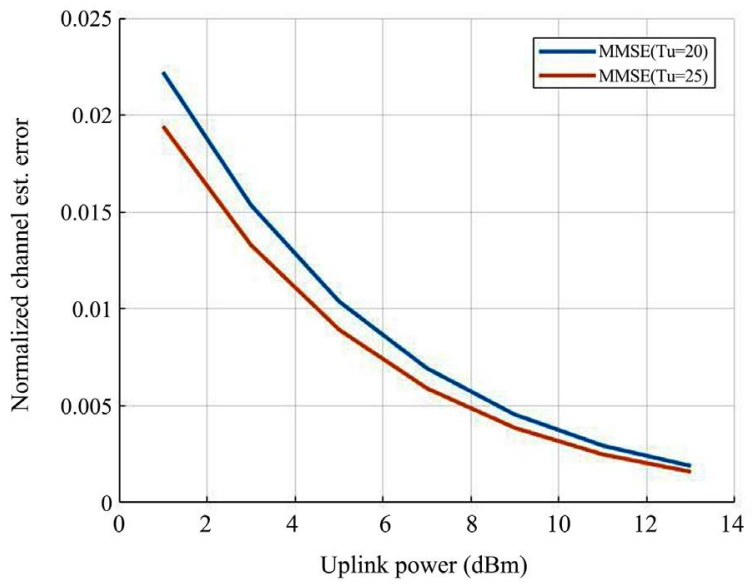

Figure 8. Performance of increasing pilot length.

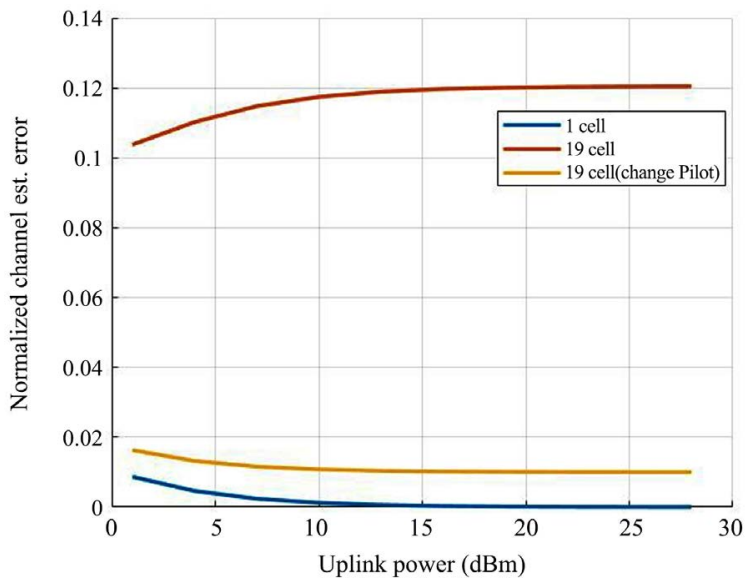

Figure 9. Performance of Method 1. 


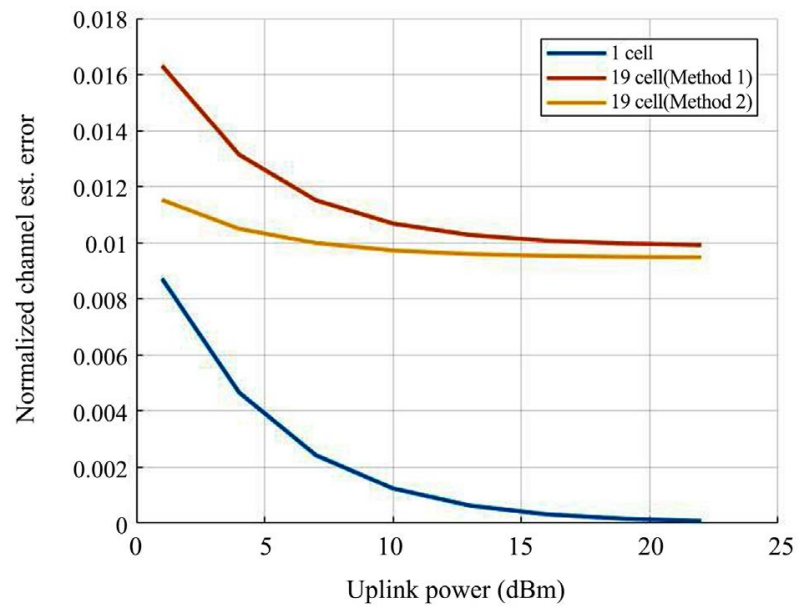

Figure 10. Comparison between Method 1 and Method 2.

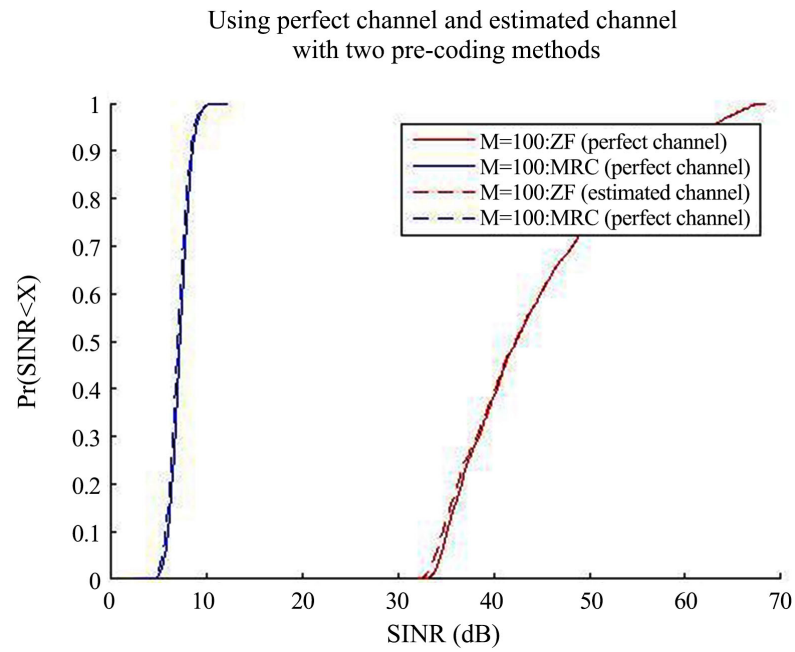

Figure 11. Using different channels with two pre-coding schemes (ZF and MRC).

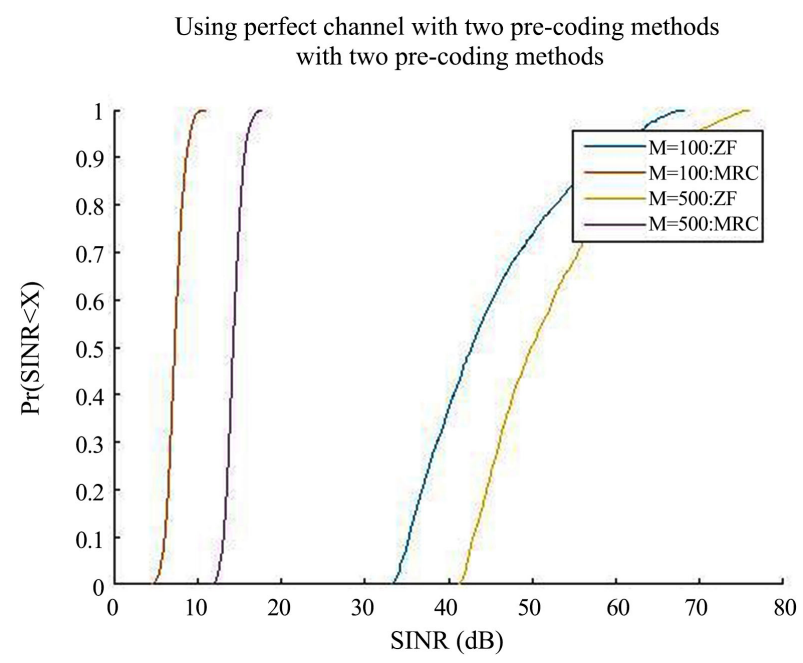

Figure 12. Using the perfect channel with two pre-coding schemes (ZF and MRC) when increasing number of antennas. 


\subsection{Performance of Power Distribution Schemes}

Figure 13 shows the performance of average SINR among all the users in the single cell. We can find that the average SINR increases when distributing power in the optimized way. This result is consistent with what we derive from the mathematical equations in Section 5.

\subsection{Impacts on Sum Rate by Changing Numbers of Antennas and Users}

From Figure 14, the data rate increases as the number of antenna increases and that is what massive MIMO aims to do. We should pay attention to the limitation that the growth rate of this curve becomes slower when there are enough antennas in the area. In other word, it's impossible for the system to achieve an infinite data rate under limited power supply.

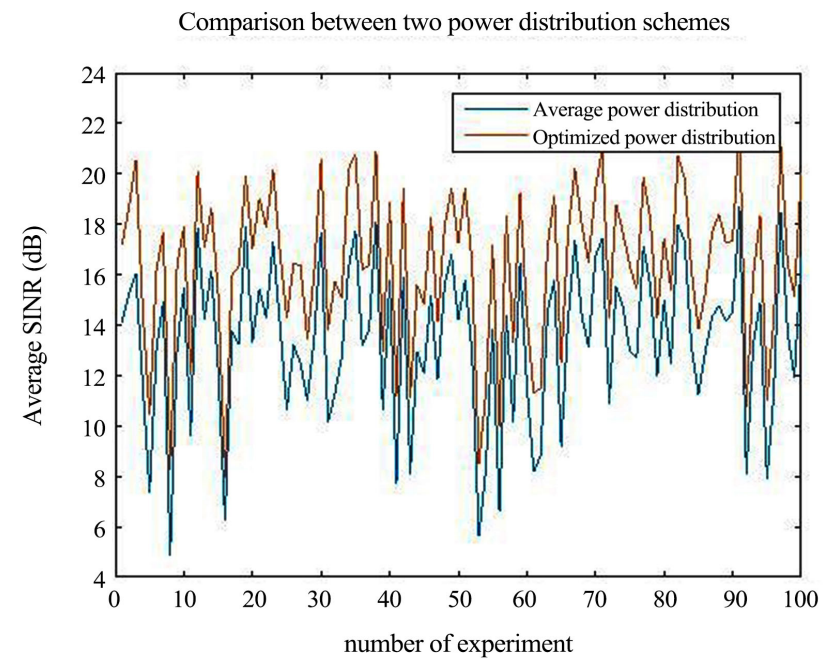

Figure 13. Comparison between two power distribution schemes (average and optimized power distribution).
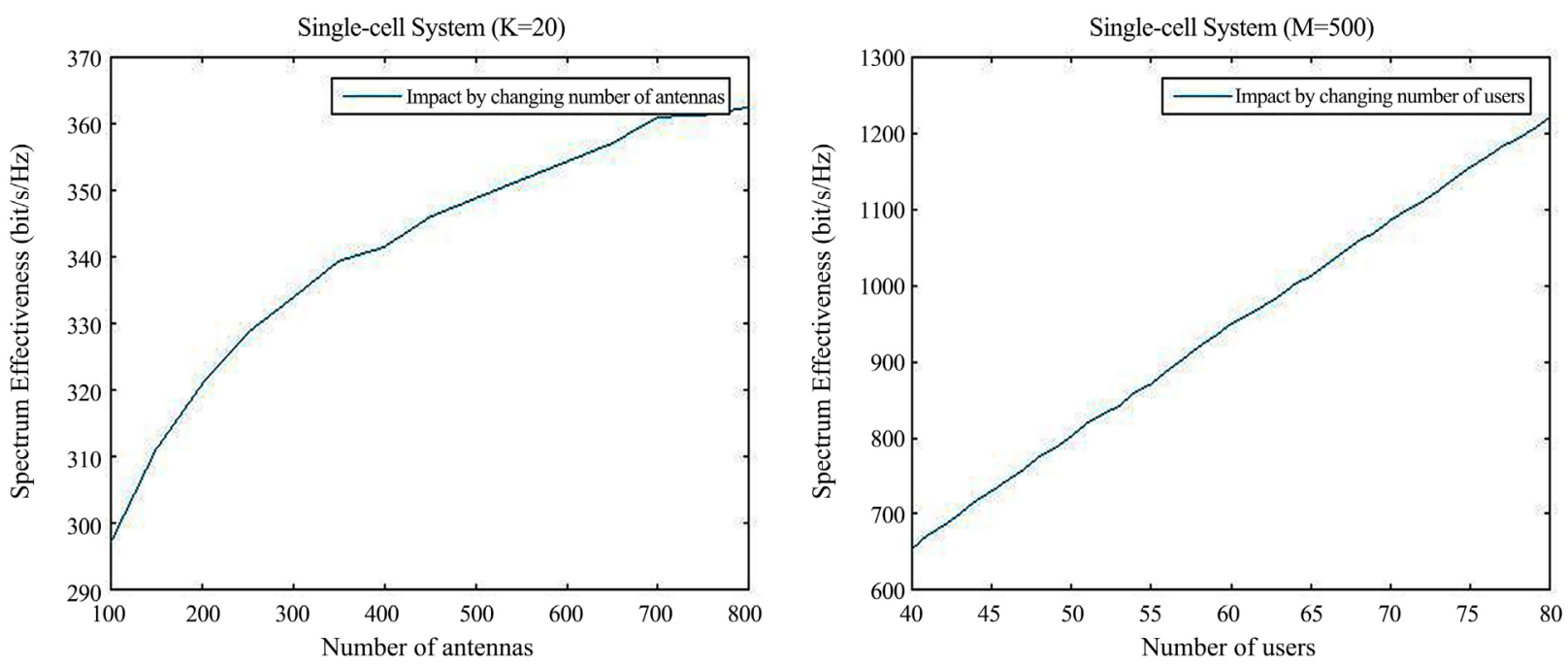

Figure 14. Changing the number of antennas and users. 
Similarly, we find that the sum data rate increases when there are more users in the system. At the meantime, the data rate for each is low if the cell contains too many users.

\subsection{Performances under Multi-Cell System}

As for multi-cell system, we run the simulation by only using the simple Zero-forcing pre-coding method of the single-cell system. In Figure 15, we can find that the system has a poor performance in multi-cell model so that we need additional complex methods to reduce the interference. This is consistent with what we analyze in last section.

\section{Discussions}

1) MMSE algorithm reduces the noise influence which are ignored in LS algorithm, so when the uplink power is low, we prefer to choose MMSE algorithm for channel estimation.

2) Longer pilot can send more power to reduce the influence of noise. So it can perform better than the short one in low uplink power situation.

3) In single-cell system, higher uplink power can reduce the influence of the noise which results in better channel estimation performance. However, in multi-cell system, it will aggravate pilot contamination from other cells so that the estimation of channel gets worse instead. We use two kinds of method to solve the problem. The second method performs better than the first because the first one only uses $1 / 3$ time of the pilot length and the second one can use all of it which could send more useful signal power. It can reduce the influence of noise as mentioned above.

4) As for pre-coding method, Zero-forcing provides better SINR than Maximal Ratio Combining does in the same situation. Therefore, we should make a trade-off between the system performance and the complexity in reality.

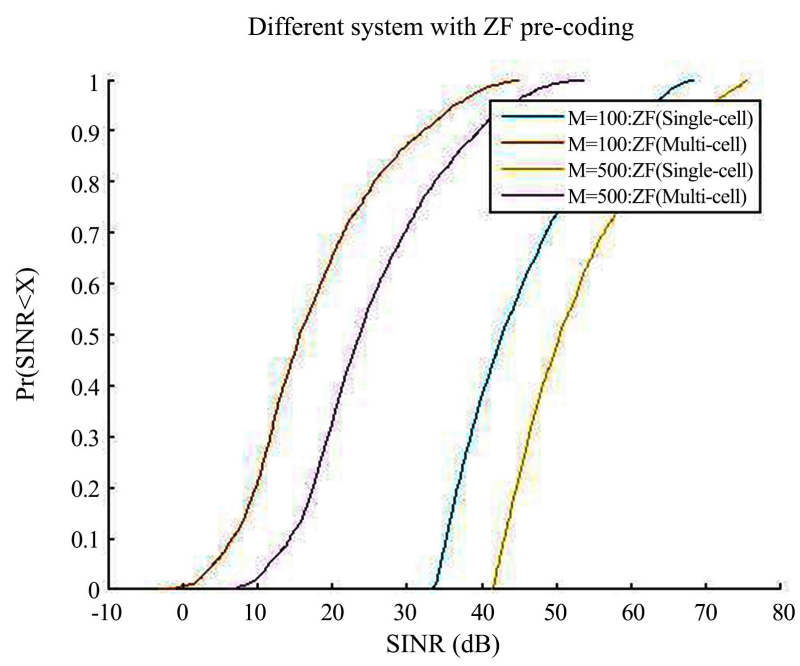

Figure 15. Downlink data transmission in multi-cell system with simple Zero-forcing pre-coding. 
5) Optimizing power distribution can improve the sum rate and the spectrum efficiency of the system. As well as this, increasing the number of antennas and users brings about similar influences. Though, like what we analyze in the previous section, it's impossible to achieve the infinite transmission rate by only changing these factors with limited power supply, we can still have these kinds of positive responses within a certain range.

\section{Conclusions and Future Work}

In this paper, we have an overview about single-cell and multi-cell massive MIMO system model, and provide some methods for channel estimation and data transmission, which overcome the pilot contamination and intra-cell interference problems. By taking in some existing methods, we develop new design for pilots and optimize algorithms for power distribution. We also find some limitations on the system capacity and propose new ideas about the prospects of this project. The performance of single-cell system is pretty good while we still need a more advanced model for multi-cell cases.

In multi-cell system, we still have some problems to solve. In channel estimation part, we only eliminate the contamination from the cells that have different types with the target cell; there is also a contamination from the same part. So we may consider a solution that if the BS have a receive power threshold $T_{s}$ and the BS will only receive the signals with power exceeds the threshold. And we design a power control factor $\gamma$ that makes the power of signals from other cells $\gamma T_{k}$ poorer than the threshold but make sure that the power of signals from target cell $\gamma T_{U}$ stronger than the threshold. In this way, the BS will only receive the signal from target cell.

In downlink data transmission part, we usually do the pre-coding at BS in target cell to reduce the interference, but sometimes the complexity is very high to do that. So now we consider a new way that each BS in the system will do the pre-coding itself in advance in order to reduce the interference to other cells. This way will largely reduce the complexity of pre-coding, but we still have a long way to prove it.

\section{Acknowledgements}

We would like to express our gratitude to all those who helped us during the writing of this paper. We gratefully acknowledge the help of our professor Danijela, who has offered us valuable suggestions in the academic studies. In the preparation of this paper, she has spent much time reading through our draft and provided us with inspiring advice. Without her patient instruction, insightful criticism and expert guidance, the completion of this paper would not have been possible. We also owe a debt of gratitude to our team member Qifeng Chen, who participated in the work of the paper. He enthusiastically collected information and discussed his thoughts with us, which sometimes gave us new ideas. Without his responsible work, we cannot get the paper done so well. 


\section{Conflicts of Interest}

The authors declare no conflicts of interest regarding the publication of this paper.

\section{References}

[1] Marzetta, T.L. (2010) NonCooperative Cellular Wireless with Unlimited Numbers of Base Station Antennas. IEEE Transactions on Wireless Communications, 9, 3590-3600. https://doi.org/10.1109/TWC.2010.092810.091092

[2] Björnson, E., Larsson, E.G. and Marzetta, T.L. (2016) Massive MIMO: Ten Myths and One Critical Question. IEEE Communications Magazine, 54, 114-123. https://doi.org/10.1109/MCOM.2016.7402270

[3] Rusek, F., Persson, D., Lau, B.K., Larsson, E.G., Marzetta, T.L. and Edfors, O. (2013) Scaling up MIMO: Opportunities and Challenges with Very Large Arrays. IEEE Signal Processing Magazine, 30, 40-60. https://doi.org/10.1109/MSP.2011.2178495

[4] Agiwal, M., Roy, A. and Saxena, N. (2016) Next Generation 5G Wireless Networks: A Comprehensive Survey. IEEE Communications Surveys \& Tutorials, 18, 1617-1655. https://doi.org/10.1109/COMST.2016.2532458

[5] Zhao, J.L. and Huo, M.M. (2017) Analysis of 5G Massive MIMO Technology. Electronic World, 24, 162+164.

[6] Wu, G.Q. (2018) Large-Scale MIMO Antenna Array Analysis for 5G. Information and Computer (Theoretical Edition), 16, 161-162.

[7] Appaiah, K., Ashikhmin, A. and Marzetta, T.L. (2010) Pilot Contamination Reduction in Multi-User TDD Systems. 2010 IEEE International Conference on Communications, Cape Town, 23-27 May 2010, 1-5. https://doi.org/10.1109/ICC.2010.5502810

[8] Wang, H.R., Wang, Y.H., Huang, Y.M. and Yang, L.X. (2013) Methods for Reducing Pilot Contamination in Massive MIMO Multi-Cell TDD System. Journal of Signal Processing, 29, 171-180.

[9] Jose, J., Ashikhmin, A., Marzetta, T.L. and Vishwanath, S. (2011) Pilot Contamination and Precoding in Multi-Cell TDD Systems. IEEE Transactions on Wireless Communications, 10, 2640-2651. https://doi.org/10.1109/TWC.2011.060711.101155

[10] Jose, J., Ashikhmin, A., Whiting, P. and Vishwanath, S. (2008) Scheduling and PreConditioning in Multi-User MIMO TDD Systems. IEEE International Conference on Communications, Beijing, 19-23 May 2008, 4100-4105.

[11] Chen, M.J. (2009). Comparative study of MMSE and LS Channel Estimation Algorithms in OFDM Systems. Journal of Sichuan University of Science and Technology (Natural Science Edition), 22, 91-93.

[12] Lu, L., Li, G.Y., Swindlehurst, A.L., Ashikhmin, A. and Zhang, R. (2014) An Overview of Massive MIMO: Benefits and Challenges. IEEE Journal of Selected Topics in Signal Processing, 8, 742-758. https://doi.org/10.1109/JSTSP.2014.2317671

[13] Zhou, Z.C., Xiao, Y. and Wang, D. (2016) Methods for Reducing Pilot Contamination in Massive MIMO Multi-Cell System. Systems Engineering and Electronics, 38, 434-441.

[14] Xie, B., Xie, S.M. and Liu, S.R. (2017) Research on Hybrid RZF Precoding Algorithm in Large Scale MIMO Systems. Journal of System Simulation, 12, 25. 